\title{
Analysis and Simulation of Contact Stresses of Convex Punch
}

\author{
Sayel M. Fayyad \\ Department of Mechanical Engineering, Faculty of Engineering Technology, \\ P.O. Box 15008, Al-Balqa Applied University Amman - Jordan
}

\begin{abstract}
This paper presents a modeling and simulation of calculating contact stresses using fractal geometry and solidworks utilities. The problem is treated in two approaches: in the first approach, the problem taking into account the existence of friction between the convex punch and the flat surface while the other by neglecting the effects of friction between the two contact surfaces with presence of heat effects. It is found that the deformation with friction is higher than the deformation without friction from numerical simulation.
\end{abstract}

Keywords:- contact stresses, convex punch, solidworks, simulation, friction, deformation.

\section{INTRODUCTION}

One of the most important task of theory of elasticity is the determination of specific pressure and deformation in the contact zone. The basic solution of contact problems was proposed by Hertz, who assumed that the surface of contacting solids is topographically smooth. Consequently the contact among solids is continuous within the nominal contact area and absent outside. This assumption excludes from consideration all real solids among which the contact is discontinuous and the real area of contact is a small fraction of the nominal contact area. When a compressive load is applied between a punch and a flat surface, the presence of surface roughness produces imperfect contact at the interface. Such contact is characterized by a large number of contact spots of various sizes spread over the whole contact interface. The degree of imperfect contact is measured by both the size of distributions of these contact spots as well as the actual area of contact, which is a fraction of the apparent or nominal surface area. The prediction of the degree of contact is of great importance to several engineering problems such as power transmission systems, belts-pulleys, gears, friction, wear, thermal and electrical contact resistance.

The topography of rough surfaces strongly influences the contact between two surfaces. Roughness measurement on a variety of surfaces has shown that their structure follows a fractal geometry whereby similar images of the surface appear under repeated magnification. Such a structure is characterized by the fractal dimension D, which lies between 2 and 3 for a surface and between 1 and 2 for a surface profile.

The contact model proposed by Borodich and Mosolov (1992) adapted as a base of this study. However the effect of the temperature on the force-deformation relationship is not studied in that work. Another study that forms a base for this work made by Abuzeid (2002).

The objective of this work is to incorporate the effect of temperature on the convex punch model proposed by Borodich and Mosolov (1992). A novel analytical thermo-elastic force-deformation relationship of a nominally convex surface is proposed. The surface will be considered as a cylindrical surface and its roughness will be modeled utilizing the fractal geometry. A power law relationship between the force applied and the approach is determined using fractal geometry analysis and Cantor structure.

The closed form solution to the contact problem was first proposed by Hertz (1892). The term Hertzian contact is often encountered in contact problems.

Early studies of the contact of rough surfaces are described in Arc hard (1957), Bowden and Tabor $(1951,964)$. The analysis of the effect of roughness on the contact interaction parameters has attracted wide attention. One of the key studies in this field was conducted by Greenwood and Williamson (1966), where the rough surface was modeled by identical spherical asperities with either exponential or Gaussian (normal) height distribution.

Greenwood and Tripp (1967) studied the elastic contact of rough spheres. A review of experimental work for the contact of rough surfaces was made by Woo and Thomas (1980). More studies were presented by Johnson (1985). These studies concluded that rough surfaces are very difficult if not impossible to create and so that surface topography is not a stationary random process and the surface parameters are related to the length of the sample. Tripp (1985) studied the Hertzian contact in two and three dimension. More recent studies was made by Liu et al. (1986), where fractal surface is constructed using the Cantor set, which is used to simulate the electrical contact properties of a rough surface interface . Chang et al. (1987) modified the original Greenwood and Williamson (1966) model to incorporate the effect of the volume conservation when asperity deforms both elastically and plastically. Several other theories of friction, wear, and lubrication based on the Greenwood and 
Williamson (1966) model were developed and discussed by Bhushan (1990). Handzel et. al (1991) made an experimental verification of the Greenwood-Williamson model for the contact of rough surfaces. However, as pointed out by Majumdar and Bhushan (1991), Majumdar et al. (1991) and Bhushan and Majumdar (1992). A new model was developed by these studies using the Weierstrass-Mandelbrot function, as described by Mandelbrot (1982), to simulate surface roughness. Borodich and Mosolov (1992) investigated the contact of a nominal flat punch using fractal geometry. The shapes of the punches were described as the Cantor-step functions and the elastic half-space was replaced by a linear Winkler foundations. An asymptotic power law which associate the force operating on the punch and the depth of indentation have been obtained, using Hill's solution (Hill, 1950) for a punch in contact with a rigid perfectly plastic half space to estimate plastic deformation.

An elastic-perfectly plastic contact of rough surfaces based on the Cantor set was modified by Warren and Krajcinovic (1994). Warren et al. (1996) studied a fractal model for the rigid-perfectly plastic contact of rough surface, where an asymptotic model was proposed, where the geometry of the rough surface is assumed to be fractal. The rough self-affine fractal structure of the effective surface is approximated using a deterministic Cantor set representation. Bhushan (1996) studied the contact mechanisms of rough surfaces in teratology single asperity contact. He also used an analytical method or solutions primarily for elastic solids and finite element solutions, primarily for elastic-plastic problems and layered solids. Yan and Kmovoipoulos (1998) studied the contact of 3D elastic plastic fractal surfaces. The main objectives of their study were to introduce comprehensive analysis of elastic-plastic rough surfaces and to present numerical results revealing the variation of the interfacial contact force and real contact area during the quasistatic surface approach, in this study also closed form solutions for the elastic and plastic components of the total normal force and real contact area are derived in terms of fractal parameters, material properties, and mean surface separation distance. Abuzeid (2002) studied the flat rough punch model and developed a relation between the force applied and approach taking into account the effect of bulk temperature. Abuzeid (2002) studied a thermo-viscous-elastic creep model for the contact of nominal flat surfaces based on fractal geometry. The contact model proposed by Borodich and Mosolov (1992) put a primitive formula to treat the convex punch in contact, without taking into account the effect of temperature. This study represents the main reference for the present work which will takes into account a new parameter that is the effect of bulk temperature. Danh T. et al.(2011), addressed the issue of the determination of the frictional stress distribution from the inversion of the measured surface displacement field for sliding interfaces between a glass lens and a rubber (poly(dimethy1siloxane)) substrate. Experimental results show that high lateral strains are achieved at the periphery of the sliding contacts. As a consequence, an accurate inversion of the displacement field requires that finite strains and non linear response of the rubber substrate are taken into account. For that purpose, a Finite Element (FE) inversion procedure is implemented where the measured displacement field is applied as a boundary condition at the upper surface of a meshed body representing the rubber substrate. Normal pressure is also determined by the same way, if non-diverging values are assumed at the contact edge.

\section{CONTACT PROBLEM}

When two bodies not mechanically Joined touch each other without becoming rigidly connected, it is said that they are in contact. They can come into contact either at a point or along a line or over a surface or a combination. Contact stresses are caused by the pressure of one solid body on another over limited areas of contact. In some cases, the contact stresses are experienced when two surfaces are pressed together by external loads. Contact stresses may be considered the major cause of failure of one body or both contacting bodies. The contact region transmits the forces from one body to the other by means of compressive and tangential or shear stresses if friction is presented. Contact-stress problems arise in the contact of wheel and a rail, in automotive valve cams and tappets ...... etc. Typical failures are seen as cracks, pits or flaking in the surface of the material. Fig 1., illustrates the cases of contact. 


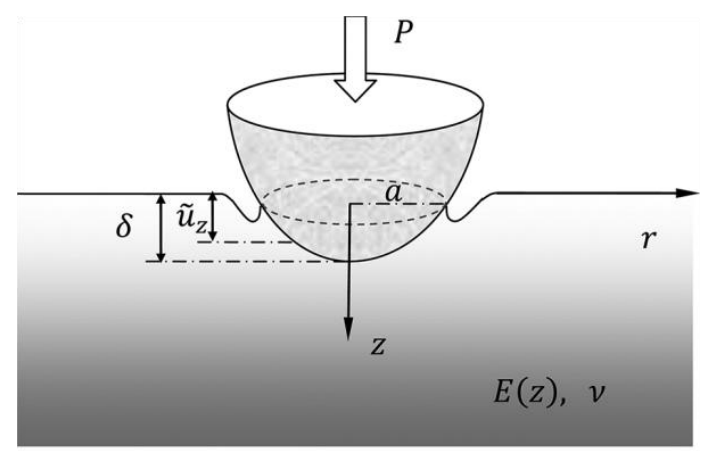

(a)

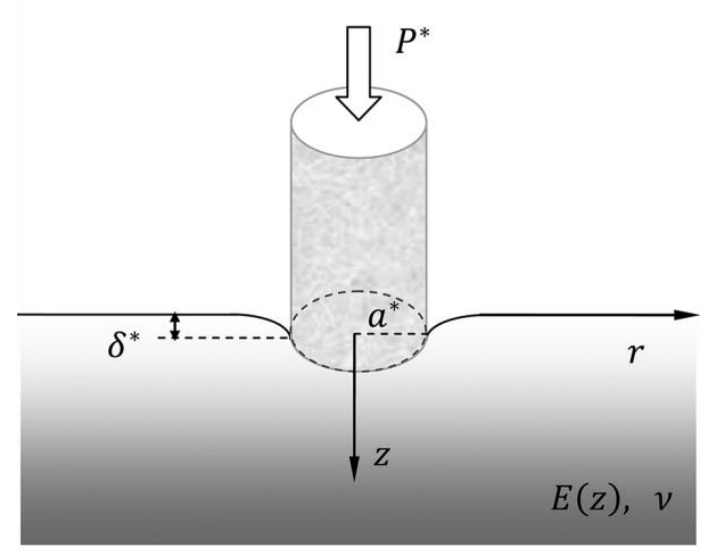

(b)

Fig. 1a,b contact problem of two surfaces.

For two cylinders in contact the maximum pressure is given by:

$\mathrm{P}_{\max }=\frac{2 \mathrm{~F}}{\pi \mathrm{bL}}$

where: $\mathrm{F}$ : force applied, $\mathrm{b}$ : width of contact area and $\mathrm{L}$ is the length of the contact area.

\section{BULK TEMPERATURE EFFECT}

In an isotropic body subject to a non-uniform temperature distribution, the elements attempt to undergo dilatation or shrinkage as a result of the changes in temperature from some initially uniform temperature. However, the elements cannot dilate or shrink in an unrestricted manner. Since the body must remain continuous during the change in temperature, there will be partial constraints as to change in geometry. We may then introduce in this way a general stress field in the body. This stress field is called thermal stress. High-Speed aircraft and space vehicles are subject to considerable thermal stresses from aerodynamic heating on the outside surfaces and from the heat originating in propulsion systems. Another example appears in the operation of spot welding between the electrodes in contact.

Thus the thermal stress in the body can be written as:

$\sigma=\frac{P}{A}=E \alpha \Delta T$

\section{THE EFFECT OF ROUGHNESS IN CONTACT PROBLEMS}

The influence of surface roughness on contact behavior is of great importance in many tribological situations. In the last decade several methods have described how to calculate the pressure distribution and the real contact area in contacts between rough surfaces. A problem arising for most contact types is that the size of the contact is much greater than the size of the asperities. Accordingly the number of contact nodes necessary for an accurate solution to the problem becomes excessively large. It is well known that surface roughness has a significant effect on how loads are transmitted at the contact interface between solid bodies. Surface roughness causes high local pressures (in the same order of magnitude as the Vickers hardness) and decreases significantly the real contact area compared to the corresponding smooth case. Apart from causing high contact stresses the surface roughness is crucial with respect to wear, friction and lubrication properties of the contact. Since the costs are significantly higher when smooth surfaces are manufactured, it is important to understand how surface roughness affects the contact conditions and to be able to predict an acceptable degree of roughness for specific contact situations, Stefan (2001). 


\section{CONVEX PUNCH MODELS}

It is well known that the parameters of the actual contact zone of real bodies depend closely on the waviness and roughness of the contact surfaces. It stands to reason that the micro geometry of the surfaces of bodies will have a large effect on their contact properties, especially at the initial stage of compression. Various contact problem for flat fractal punches were considered in Borodich and Mosolov (1992), warren and Krajcinovic (1995) and Warren, Majumdar and Krajcinovic (1996) and Abuzaid (2002). The shapes of the punches were described as the Cantor-step functions and the elastic half-space was replaced by a linear Winkler foundation. Asymptotic power laws which associate the force operating on the punch and the depth of indentation have been obtained. But, for convex punches only little work had been done to study this kind of contact stresses. In this work we will develop a new model for a rough convex punch depending on Mosolov, and Borodich (1992) studies and Abuzeid (2002) taking into account the effect of temperature as an extra load.

- Analysis of Convex Rough Punch Contact Model.

For a convex punch, consider the profile function of the surface such that $\mathrm{Z}=\mathrm{f}(\mathrm{x})$, Mosolov and Borodich (1992) having a fractal surface. Let (u) be the depth of indentation (approach) of the point of the punch under load. It is also assumed that the profile of the punch is symmetrical about the Z-axis. The contact pressure under the punch at any point ( of the punch) depends only on its displacement $(\mathrm{u})$. The contact force $(\mathrm{P})$ along the convex punch is given by the formula of Mosolov and Borodich (1992):

$$
P=\int_{0}^{L_{O}} P(x) d x, \quad f\left(L_{O}\right)=u
$$

Where $P(\mathrm{x})$ can be given as:

$$
P(x)=C[u-f(x)]^{\gamma}
$$

Depending on [28] the force-displacement relation can be written as

$$
p^{*}=\left[\frac{E}{8 b(1+\chi)}\left(u^{*-1}\right)^{\gamma+\frac{1}{2}}-\frac{E \alpha \Delta}{2}\left(u^{*}\right)^{\gamma+\frac{1}{2}}\right] \beta\left(\frac{1}{2}, \gamma+1\right)
$$

Where E: Modulus of Elasticity, $\square \square$ : Thermal Coefficient Expansion and $\square$ : bulk Temperature change, $\beta(x, y):$ Beta function, $b$ : width of contact area and $L$ is the length of the contact area, $\square \square=\frac{l n a}{\ln b}$ and

$$
\gamma=\frac{2-D_{F}}{1+D c-D_{F}}
$$

Equation (5) represents the relationship between the force applied and the approach for the convex punch model in a non dimensional form taking into account the effect of the bulk temperature.

\section{MODELING AND SIMULATION: RESULTS AND DISCUSSION}

Figure 2 shows the model information of the contact stresses using solid works simulation without friction.

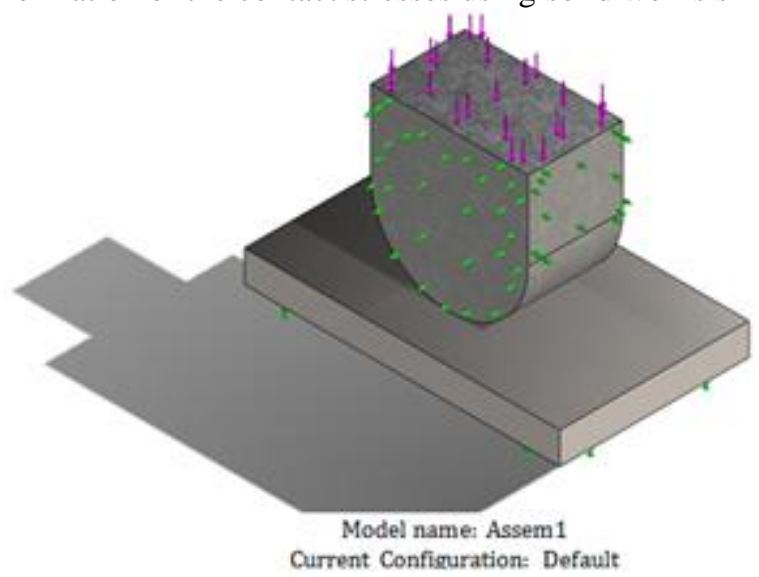

Fig.2contact stress model simulation 
Figure 3 shows the model information

\begin{tabular}{|c|c|c|c|}
\hline & & & Solid Bodies \\
\hline $\begin{array}{c}\text { Document Name and } \\
\text { Reference }\end{array}$ & Treated As & Volumetric Properties & $\begin{array}{c}\text { Document } \\
\text { Path/Date Modified }\end{array}$ \\
\hline Boss-Extrude1 & Solid Body & $\begin{array}{c}\text { Mass:526.916 kg } \\
\text { Volume:0.0669524 m^3 } \\
\text { Density:7870 kg/m^3} \\
\text { Weight: } 5163.77 \mathrm{~N}\end{array}$ & $\begin{array}{c}\text { C: } \backslash \text { Users } \backslash \text { Mohd } \backslash \text { Do } \\
\text { cuments } \backslash \text { Dr.sayel } \\
\text { project } \\
2 \backslash \text { Part1.SLDPRT } \\
\text { Nov } 2615: 59: 57 \\
2012\end{array}$ \\
\hline Boss-Extrude1 & Solid Body & $\begin{array}{c}\text { Mass: } 462 \mathrm{~kg} \\
\text { Volume: } 0.06 \mathrm{~m}^{\wedge} 3 \\
\text { Density: } 7700 \mathrm{~kg} / \mathrm{m}^{\wedge} 3 \\
\text { Weight: } 4527.6 \mathrm{~N}\end{array}$ & $\begin{array}{c}\text { C: \Users } \backslash \text { Mohd } \backslash \text { Do } \\
\text { cuments } \backslash \text { Dr.sayel } \\
\text { project } \\
\text { 2\Part2.SLDPRT } \\
\text { Nov } 2616: 00: 57 \\
2012\end{array}$ \\
\hline
\end{tabular}

Fig. 3 model information

Figure 4 shows the contact bodies meshing.

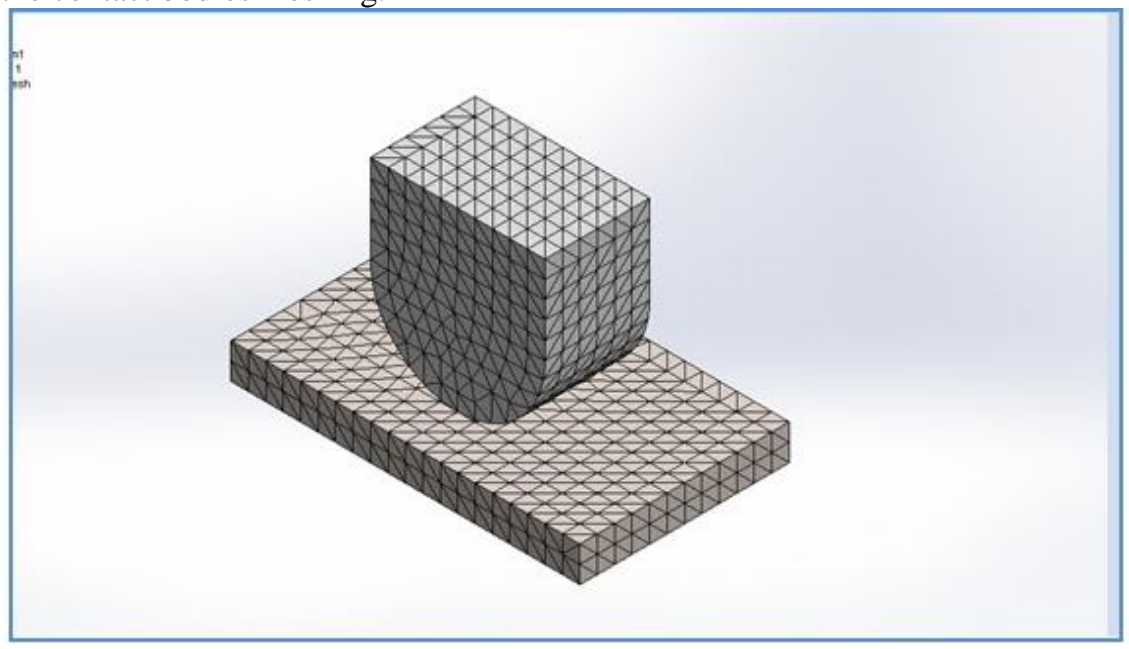

Fig. 4 meshing of two contact bodies

Figure 5 shows the von-Misses stress distribution of the contacted bodies.

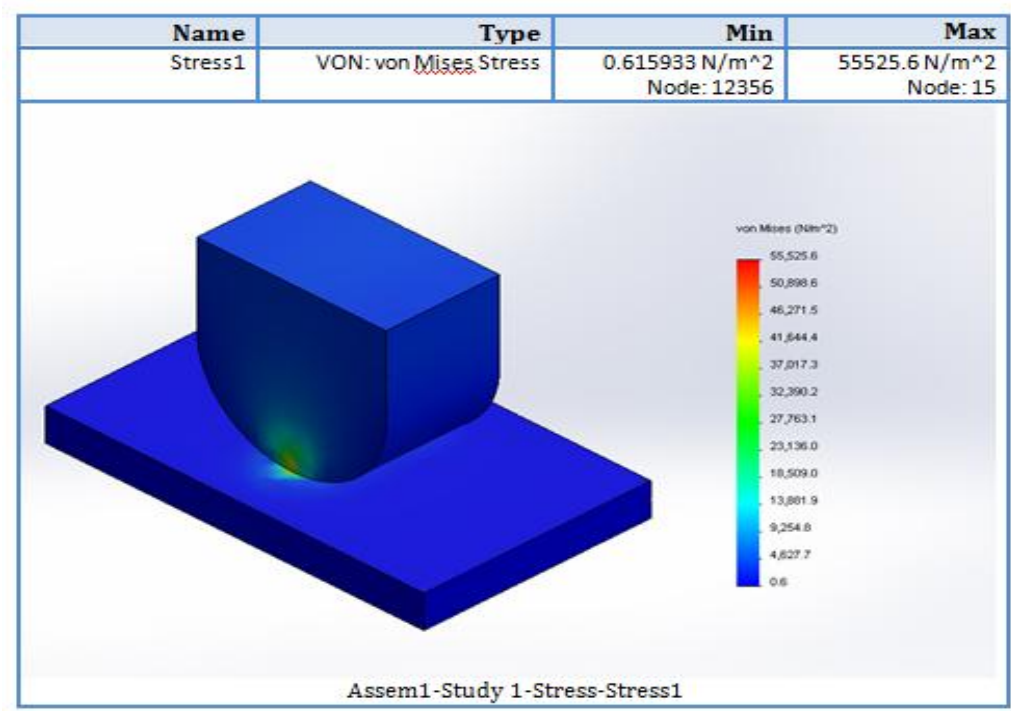

Fig.5 von-Misses stress distribution

Figure 6 shows the displacement or deformation of the two contacted bodies 


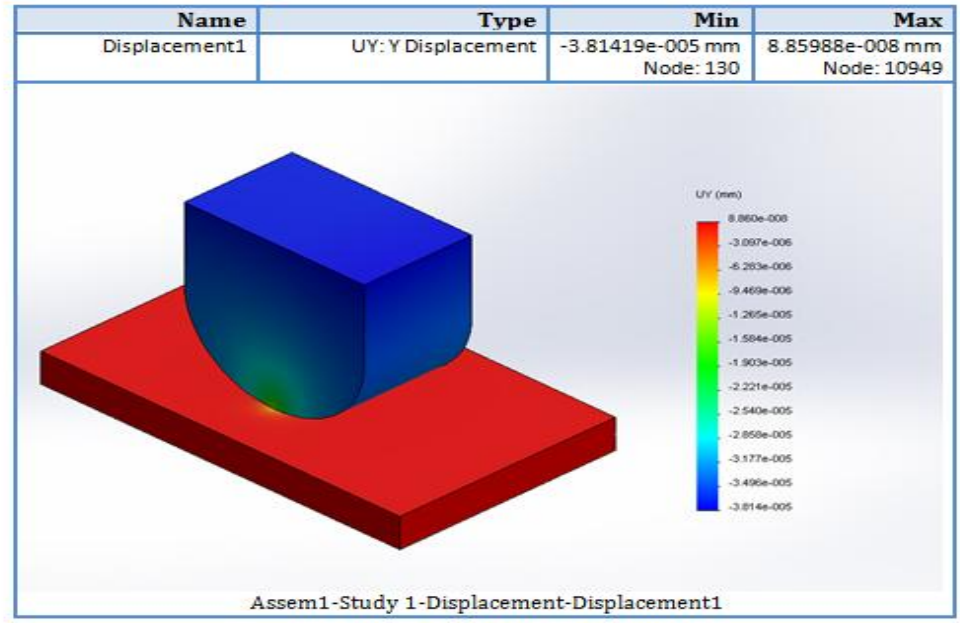

Fig. 6 displacement distribution

Figure 7 shows the strain distribution of the two contacted bodies.

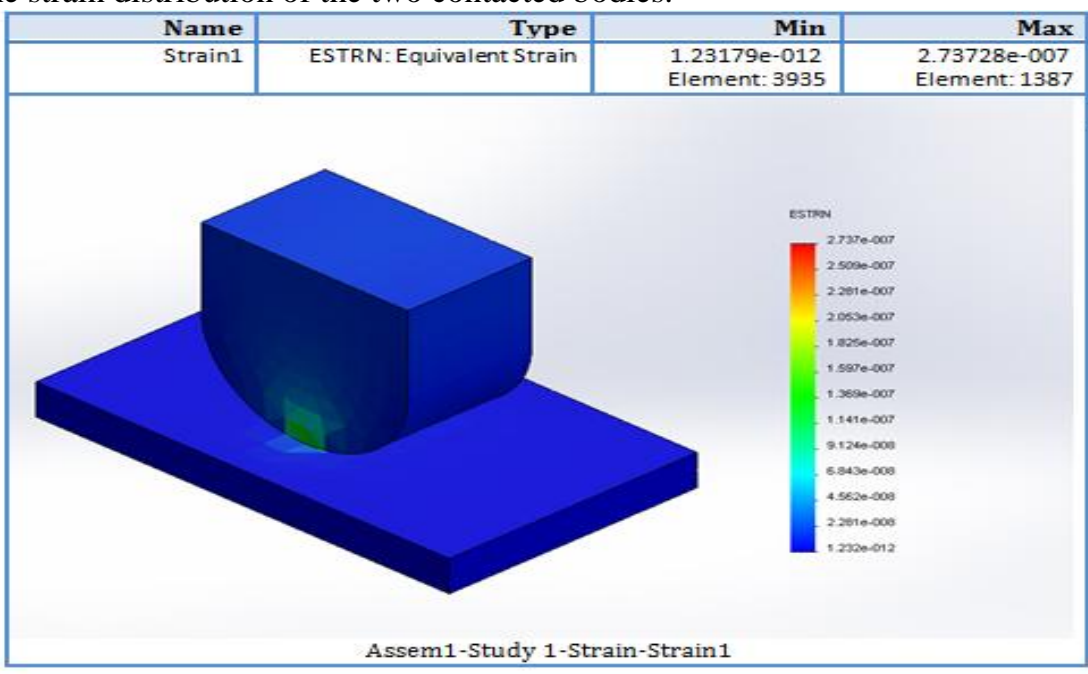

Fig. 7 strain distribution

Figure 8 shows the total or resultant displacement of the contacted bodies.

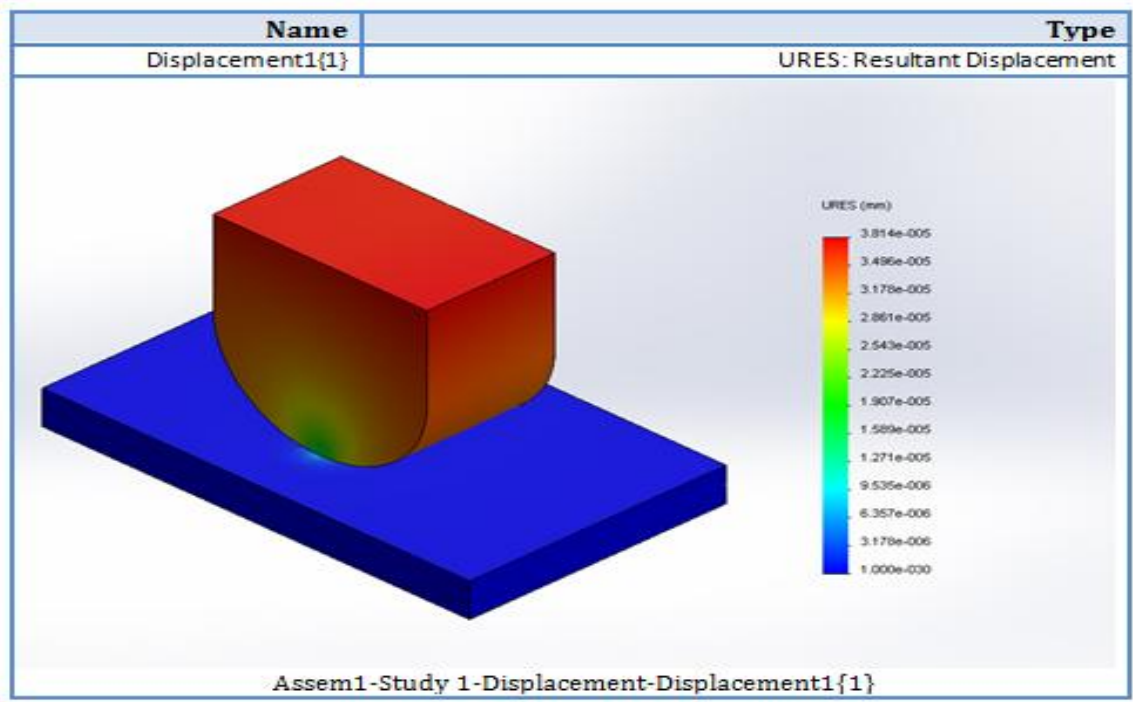

Figure 8 resultant displacements.

Figure 9 shows the stress distribution of the two contact bodies with friction. 


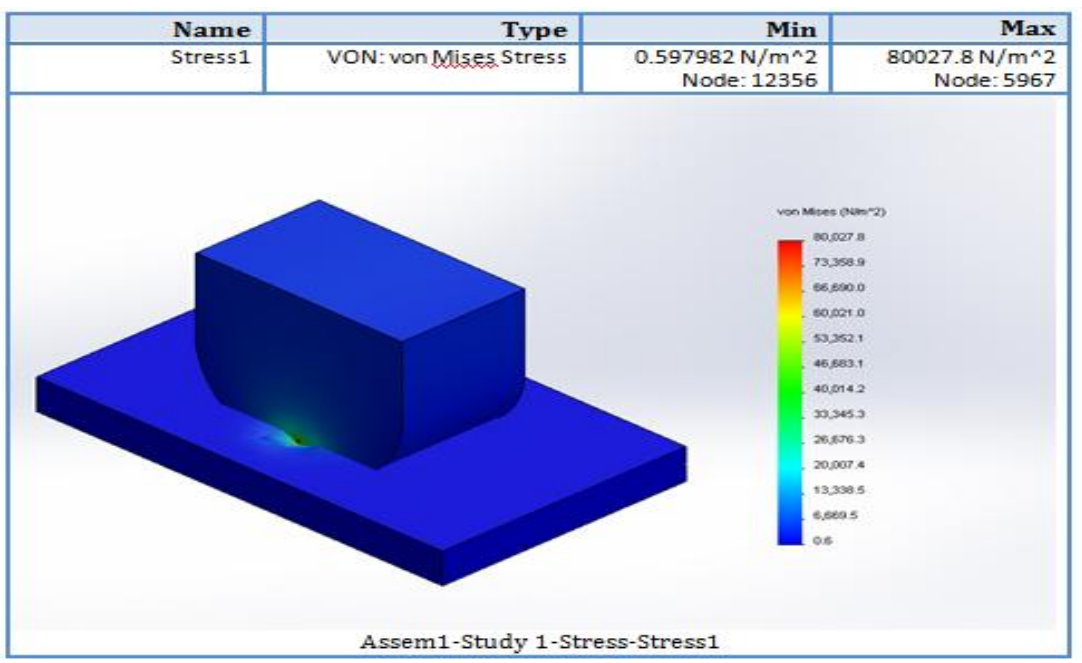

Figure 9 Von-Misses stress distribution

Figure 10 shows the displacement of the two contacted bodies with friction.

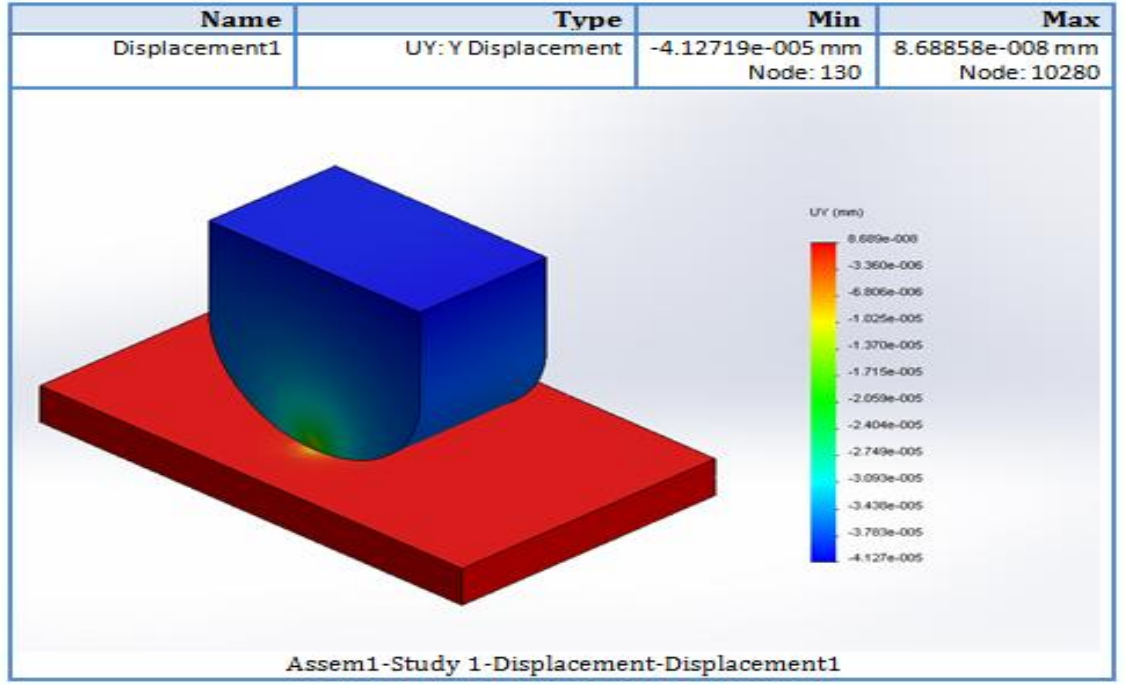

Fig. 10 the displacement distribution of the contacted bodies

Figure 11 shows the strain distribution of the two bodies in contact.

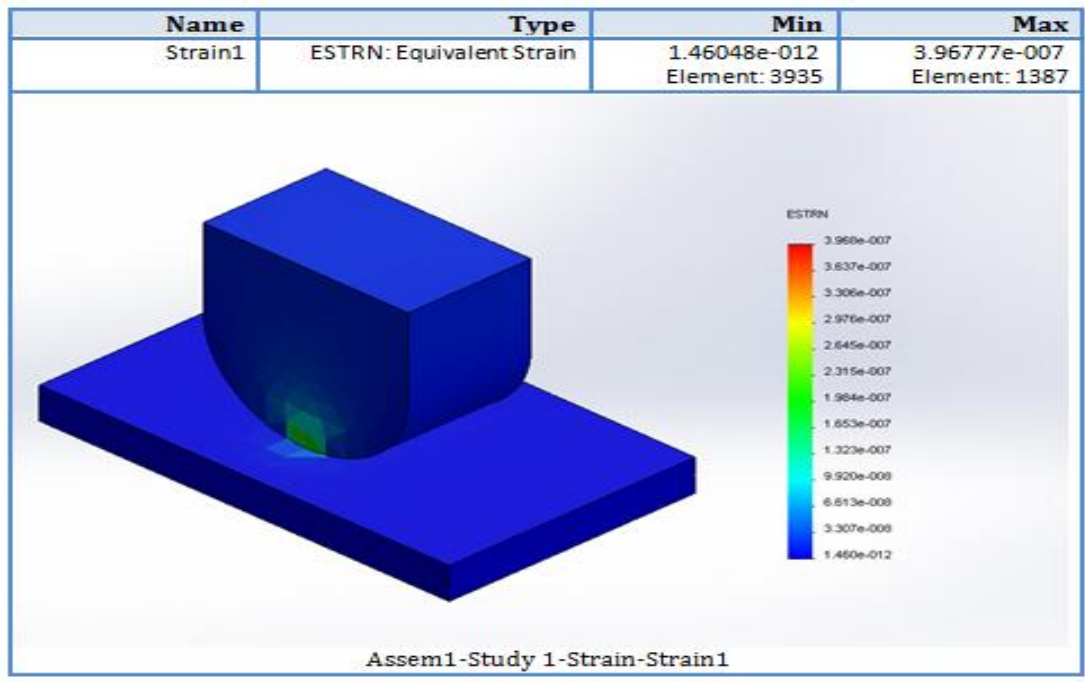

Fig. 11 strain distribution of the two contacted bodies.

Figure 12shows the total or resultant displacement of the contacted bodies with friction. 


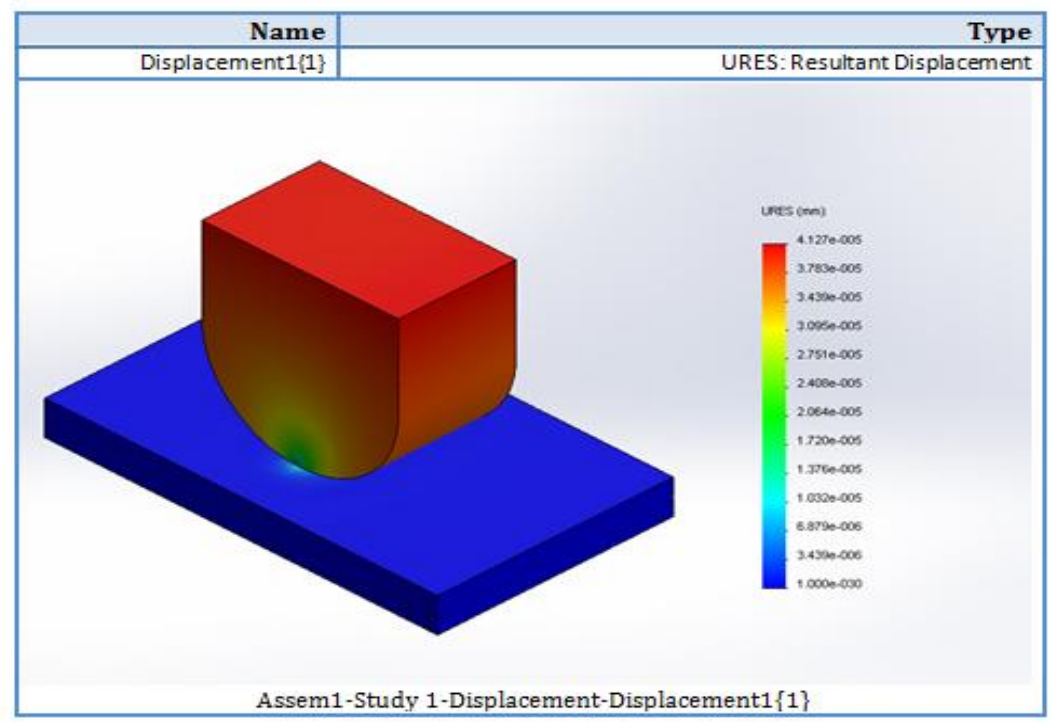

Fig. 12 total or resultant displacement of the two contacted bodies.

\section{CONCLUSION}

Equation 5 obtained can be used to conduct exact analytic investigations of the solutions of contact problem of the convex punches regardless of their radius. But, nevertheless, it might turn out to be not too far from the reality, since tests on the actual contact area of polished ( ground ) metal surface show they contain sets of parallel ragged-edged scratches of different depths .

It has been shown that the effect of bulk temperature on the relationship between the applied force and the approach is small which can be neglected, and if the contact problem is self-similar then its properties are independent of the choice of boundary conditions and valid for linear and nonlinear, isotropic and anisotropic media. So, we can say that the self-similar law of change of the solution is the general property of the considered problems.

The friction has a considerable effect on the displacement of the contact area, the displacement with the existence of friction is more than that without the existence of friction.

\section{REFERENCES}

[1]. Abuzeid, O. M 2002. Fractal Model of a Linear Thermoelastic Contact Between Flat Rough Surfaces Based on Cantor Structure. Sixth International conference on production \& design for development- PEDD6, Cairo.

[2]. Abuzeid, O. M. 1997. Theoretical Analysis and Numerical Applications of the Contact Between Two Elastic Bodies Under Compression. Ph.D. Thesis, Politecnico di Torino, Italy.

[3]. Abuzeid, O. M. 2002. A linear Thermo-Visco-Elastic Creep Model for the Contact of Nominal Surfaces Based on Fractal Geometry :Kelvin-Voigt Medium. In t.J. Solides Structure, 25(1): 25-30.

[4]. Abuzeid, O. M. 2003. Linear Visco-Elastic Creep Model for the Contact of the Nominal Surfaces Based on Fractal Geometry: Maxwell type medium. Dirasat, Engineering Sciences, 30(1): 30-35.

[5]. Arthur, P. B. and Omar, M. S. Advance Mechanics of Materials, $4^{\text {th }}$ edition. Plenum press, New York, USA.

[6]. Bharat, B. 1996. Contact Mechanics of Rough Surfaces in Tribology: Single Asperity Contact. Applied Mechanics J 49 (5): $110-120$.

[7]. Borodich, F. M. and Mosolov, A. B. 1992. Fractal Roughness in Contact Problems. J. Appl. Maths. Mechs, 56 (5): 681-690.

[8]. Borodich F. M. 1993. The Hertz Frictional Contact Between Nonlinear Elastic Anisottropic Bodies (the similarity approach). Int. J. Solids Structure, 30 (11): 1512-1526.

[9]. Borodich F. M. 1993. Similarity Properties of Discrete Contact Between a Fractal Punch and Elastic Medium. Mechanics of Solids, t. 316, Seris II: 281-286.

[10]. Feder, J. 1988. Fractals, $2^{\text {nd }}$ edition. Plenum press, New York, USA.

[11]. Greenwood, J. A. and Tripp, J. H. 1967. The Elastic Contact of Rough Spheres. Journal of Applied Mechanics, March/153.

[12]. Handzel, Z. T. and Polijaniuk, A. 1992. On Experimental Verification of the Greenwood-Williamson model for the contact of rough surfaces. Wear, 154: 115-124.

[13]. Hearn, E. J. 1997. Mechanics of Materials, $3^{\text {rd }}$ ed. Cambridge University, Britain.

[14]. Hughes, J. R. and Marsden, E. J. 1983. Mathematical Foundations of Elasticity. Plenum Press, USA.

[15]. John, H. T. 1985. Hertz in Contact in Two and Three Dimension. NASA Technical Paper, 2473.

[16]. John, C. R. 1994. Fractal Surfaces. Plenum press, New York.

[17]. Johnson, K. L. 1985. Contact Mechanics. Cambridge University Press, England. 
[18]. Majumdar, A. and Tien, C. L. 1991. Fractal network model for contact conductance. Journal of Heat Transfer, 516(113) : 628-634.

[19]. Meguid, S. A. et. al. 2001. Numerical and Experimental Studies of Multiple Discs in Contact.International journal of computational engineering science, 2(1): 75-93.

[20]. Miroslav, M. N. 1995. Fractal Reviews in the Natural and Applied Sciences. Chapman \& Hall, Britain.

[21]. Panagiotopoulos, P. D. 1991. Fractal Geometry in Solids and Structure. Int. J. Solids Structures, 29(17): 2159-2175.

[22]. Panagiotopoulos, P. D. and Panagouli, O. K. 1994. Numerical Methodes for Convex Energy Problems. Int. J. Solids Structures, 31(16): 2211-2228.

[23]. Panagiotopoulos, P. D. et. al. 1993. Fractal Geometry and fractal Material Behavior in Solids and Structures. Archive of Applied Mechanics, 63(5): 1-24.

[24]. Robert, C. W. Handbook of Tables for Mathematics, $4^{\text {th }}$ edition. The Chemical Rubber CO, England.

[25]. Robert, D. C. and Warren, C. Y. 1985. Advance Mechanics of Materials, Macmilliam, USA.

[26]. Rubtsov, V. E. et. al. 1998. Study of the Formation of Contact Between Rough Surfaces on the Particle Method. Technical Physics Letters, 24(3): 110-120.

[27]. Stefan, B. 2001. The Influence of Surface Roughness in Elliptical Contacts. Tribology International J, 34 (5): 841 845.

[28]. Sayel M.F., Osamah A.Z., 2003, "Thermelastic contact model to predict the deformation of convex punch using fractal geometry". Master thesis-UJ.

[29]. Takayasu, H. 1990. Fractals in the Physical Science. Manchester University Press, England.

[30]. Takayasu, H. 1990. Fractals in the physical Science. MacMilliam, New York, USA.

[31]. Thomas, L. W. and Dusan, K. 1995. Fractal Models of Elastic-Perfectly Plastic Contact of Rough Surfaces Based on the Cantor Set. International Journal of Solids Structure, 32(19): 2907-2922.

[32]. Warren, T. L. et. al. 1996. A Fractal Model for the Rigid Perfectly Plastic of Rough Surfaces. Journal of Applied Mechanics, 63(7): 47-54.

[33]. Woo, K. L. and Thomas, T.R. 1980. Contacts of Rough Surfaces: A review of Experimental work. Wear, 58(5): 331340.

[34]. Yan, W. and Komvopoulos, K. 1988. Contact Analysis of Elastic-Plastic Fractal Surfaces. Journal of Applied Physics, 84(7): 3617-3624.

[35]. Danh Toan Nguyen, Pierdomenico Paolino1, M.C. Audry, Antoine Chateauminois, Christian Fretigny, Yohan Le Chenadec, M. Portigliatti2, E. Barthel, 2011, "SURFACE PRESSURE AND SHEAR STRESS FIELDS WITHIN A FRICTIONAL CONTACT ON RUBBER", The Journal of Adhesion 87, 3 (2011) 235-250". DOI : 10.1080/00218464.2011.557340. 